Int. J. Contemp. Math. Sci., Vol. 2, 2007, no. 1, 1 - 11

\title{
On Ruled Surfaces in Euclidean Space $\mathbf{E}^{3}$
}

\author{
Ayhan Sarıŏlugil and Ayhan Tutar \\ Department of Mathematics, Faculty of Science and Arts \\ Ondokuz University, 55139-Kurupelit, Samsun, Turkey \\ ayhans@omu.edu.tr, atutar@omu.edu.tr
}

\begin{abstract}
The main goal of this paper, some characteristic properties of a ruled surface in $E^{3}$ are given related to the geodesic curvature and the second fundamental form of its.
\end{abstract}

Mathematics Subject Classification: 53A05, 53A15

Keywords: Ruled surface, Geodesic curvature, Striction line, drall

\section{INTRODUCTION}

Ruled surfaces were investigated by G.Monge, who established the partial differential equation satisfies by all ruled surfaces. Thus, ruled surfaces are formed by one-parameter set of lines and have been investigated by V. Hlavaty [26] and J. Hoschek [20].

Furthermore, the theory of ruled surfaces has been applied to kinematics. Especially, the study of one-parameter closed motions became an interesting subject in kinematics after the work of J. Steiner [19] and A. Holditch [1].

During the second half the nineteenth century there were appeared many publications about Steiner and Holditch theorems, for example: A. B. Kempe [2], [3], [4] and [5], C. Leudesdorf [17] and [18], E. B. Elliot [9], H. Frank [18], H. R. Müller [11] and [12], H. Potmann[14] and [15], O. Bottema [23], R. Thüring [25] and J.Tölke [21] etc.

Elliot wrote about the spherical motions whereas the others wrote only on planar. In 1948, W. Blaschke defined the Steiner point and Steiner vector for one-parameter closed spherical motions,[27]. H. R. Müller showed that the pitch of a closed ruled surfaces in are simple geometrical integral invariants, [13]. Recently, several authors have used the this invariants in their investigations concerning the generalization of some theorems of the theory ruled surfaces, for example: O. Giering [24], C. Thas [8], H. H. Hacısalihoğlu [16], N. Kuruoğlu and S. Keleş [22] etc. 
Finally, in this paper we are give some characteristic properties the ruled surfaces related to the geodesic curvature and second fundamental form of its.

\section{Basic Concepts}

In this section we will give some basic concepts related to the theory of surfaces and curves in for later use.

Let $M$ be an regular oriented surface with unit normal vector $N$ in 3-dimensional Euclidean space $E^{3}$. The shape operator on $M$ is a linear transformation

$$
S_{P}: T_{M}(P) \rightarrow T_{M}(P)
$$

defined by

$$
S_{P}(X)=D_{X}(N)
$$

where $D$ is affine connnection on $E^{3}$ and

where is affine connection on , [10]. The symmetric bilinear function

$$
I I_{P}: T_{M}(P) \times T_{M}(P) \rightarrow I R
$$

defined by

$$
I I_{P}(X, Y)=\left\langle S_{P}(X), Y\right\rangle
$$

where $X, Y \in T_{M}(P),[10]$.

Let $\alpha$ be a curve with parametrized by arc-length $s$ on the surface $M$ in $E^{3}$. For the covariant derivate formulas of the Frenet frame $\left\{V_{1}, V_{2}, V_{3}\right\}$ of the curve $\alpha$ on $M$ can be written

$$
\left[\begin{array}{c}
\dot{V}_{1} \\
\dot{V}_{2} \\
\dot{V}_{3}
\end{array}\right]=\left[\begin{array}{ccc}
0 & k_{1} & 0 \\
-k_{1} & 0 & k_{2} \\
0 & -k_{2} & 0
\end{array}\right]\left[\begin{array}{l}
V_{1} \\
V_{2} \\
V_{3}
\end{array}\right]
$$

where $k_{1}$ and $k_{2}$ are the curvature and torsion of the curve $\alpha,[17]$.

Similarly, for the covariant derivate formulas of the Darboux frame $\left\{E_{1}, E_{2}, N\right\}$ of the curve $\alpha$ on $M$ can be written as

$$
\left[\begin{array}{c}
\frac{d E_{1}}{d s} \\
\frac{d E_{2}}{d s} \\
\frac{d N}{d s}
\end{array}\right]=\left[\begin{array}{ccc}
0 & k_{g} & I I\left(E_{1}, E_{1}\right) \\
-k_{g} & 0 & I I\left(E_{1}, E_{2}\right) \\
-I I\left(E_{1}, E_{1}\right) & -I I\left(E_{2}, E_{2}\right) & 0
\end{array}\right]\left[\begin{array}{c}
E_{1} \\
E_{2} \\
N
\end{array}\right]
$$

where $k_{g}$ is the geodesic curvature,[10]. 


\section{On Ruled Surfaces in $E^{3}$}

In this section, some characteristic properties of ruled surface in $E^{3}$ are given related to the geodesic curvature $k_{g}$ and the second fundamental form II of its, respectively.

As is well known, a ruled surface is two - dimensional manifold of such that through each point in there passes a segment a straight line which lies in

Let $I \subset I R$ be an interval, let $\alpha: I \rightarrow E^{3}$ be a regular parametrized curve, and let $\mathrm{X}: I \rightarrow E^{3}$ be an arbitrary smooth function with $X(s) \neq \overrightarrow{0}$ for all $s \in I$. Thus, we defined a parametrized surface by

$$
\varphi(s, v)=\alpha(s)+v X(s), s \in I, v \in I R,
$$

This is called a ruled surface with rulings $X$ and directrix $\alpha$.

Theorem 1. : Let be a ruled surface in. The rulings of are both asymptotic and geodesic.

Proof. : The proof is clear.

We study the neighbourhood of a point $P$ in a ruled surface $M$. We assume that the ruling $X(s)$ is orthogonal to the directrix $\alpha$ at each point. Thus, we can write $\langle T, X\rangle=0$ where $T$ is the tangent vector field of the directrix $\alpha$. Let $N$ be a local unit normal for this coordinate neighbourhood. Thus, the unit vector fields $T, X, N$ give orthonormal frame along $\alpha$, and we next obtain the Frenet formulas for this frame. On $\alpha$ we have $\langle T, T\rangle=\langle X, X\rangle=\langle N, N\rangle=1$ so $0=T[\langle X, X\rangle]=\left\langle D_{T} X, X\right\rangle$ implies normal $D_{T} X$ to $X$. Similarly, $D_{T} N$ normal to $N$ and $D_{T} T$ normal to $\mathrm{T}$. From here we can write as

$$
\left[\begin{array}{c}
D_{T} T \\
D_{T} X \\
D_{T} N
\end{array}\right]=\left[\begin{array}{ccc}
0 & k_{g} & I I(T, T) \\
-k_{g} & 0 & I I(T, X) \\
-I I(T, T) & -I I(T, X) & 0
\end{array}\right]\left[\begin{array}{c}
T \\
X \\
N
\end{array}\right]
$$

where $k_{g}$ and $I I$ are the geodesic curvature and the second fundamental form of , respectively. Holding $v$ constants, we get a curve $\varphi_{v}(s)=\alpha(s)+v X(s)$ on $M$ with tangent

$$
A=\left(1-v k_{g}\right) T+v I I(T, X) N
$$

From here, we can say that the vector field $A$ is normal to $X$. Thus, the following theorem can be given.

Theorem 2. : Let $M$ be a ruled surface in $E^{3}$. The tangent planes of $M$ along a ruling are constants if and only if $I I(T, X)=0$.

Proof. :The tangent space along a ruling is spanned by $A$ and $X$ ( and $A$ is orthogonal to $X$ ), hence this tangent space is constant along a ruling. Then, the vector field $N$ is constant along a 


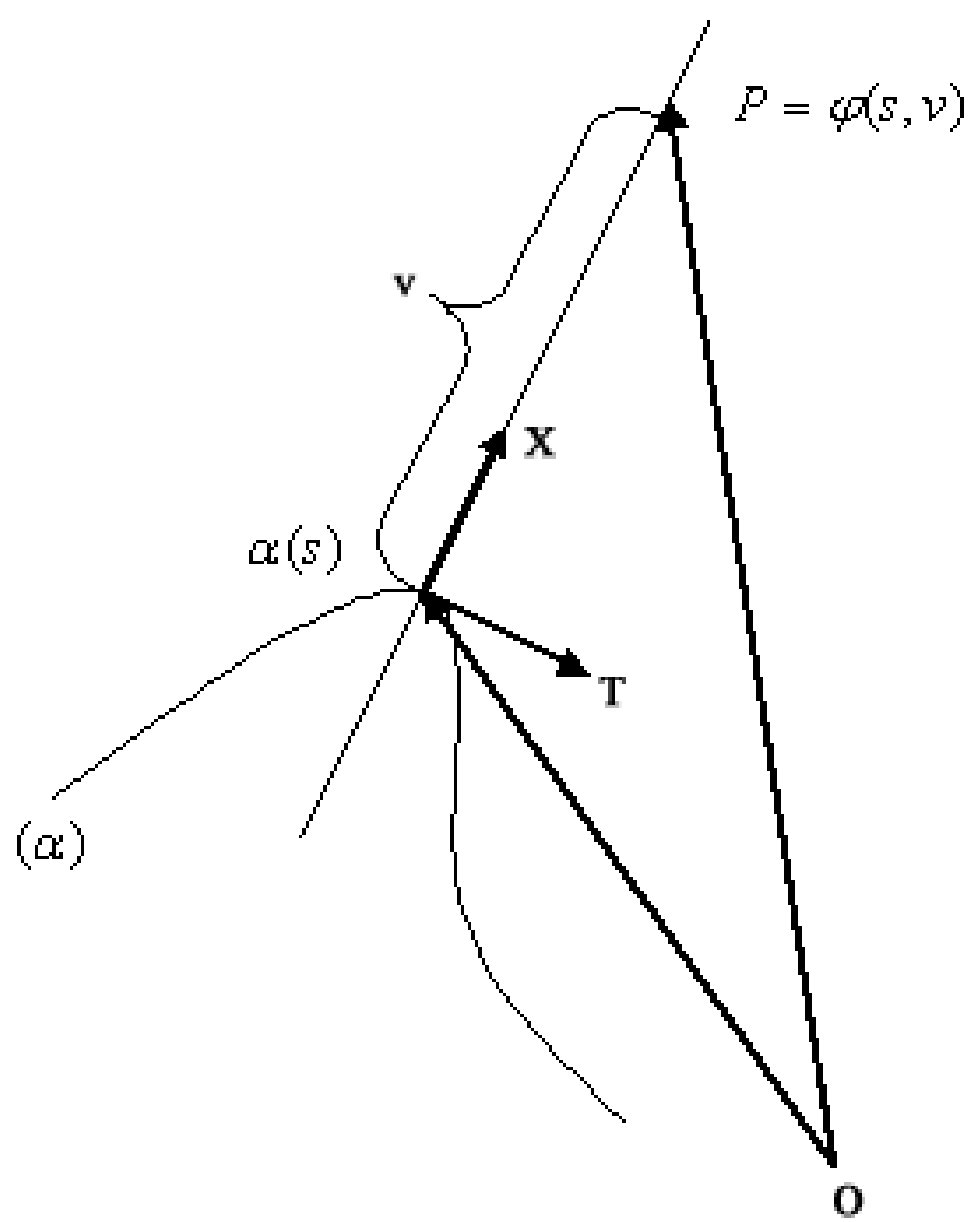

Figure 1

ruling $\alpha$. Thus, the system $\{A, T\}$ is linear dependent. By the eq. (3.3), we get $I I(T, X)=0$. Then, tangent planes of $M$ along a ruling is constant iff $I I(T, X)=0$.

This is complete the proof.

Definition 1. : Let $M$ be a ruled surface in $E^{3}$. The ratio the shortest between the two neighbouring rulings to the angle between this rulings is called the distribution parameter(or drall) of the ruled surface $M$.

Now, we will compute the distribution parameter (or drall) of the ruled surface $M$. Let $P_{X}$ be the distribution parameter of the ruling $X$. We assume that the two neighbouring rulings are $X$ and $X+d X$. Then the vector $X$ $\Lambda d X$ is orthogonal to the each vectors $X$ and $X+d X$.

For the unit vector direction the vector $X \Lambda d X$ we have 


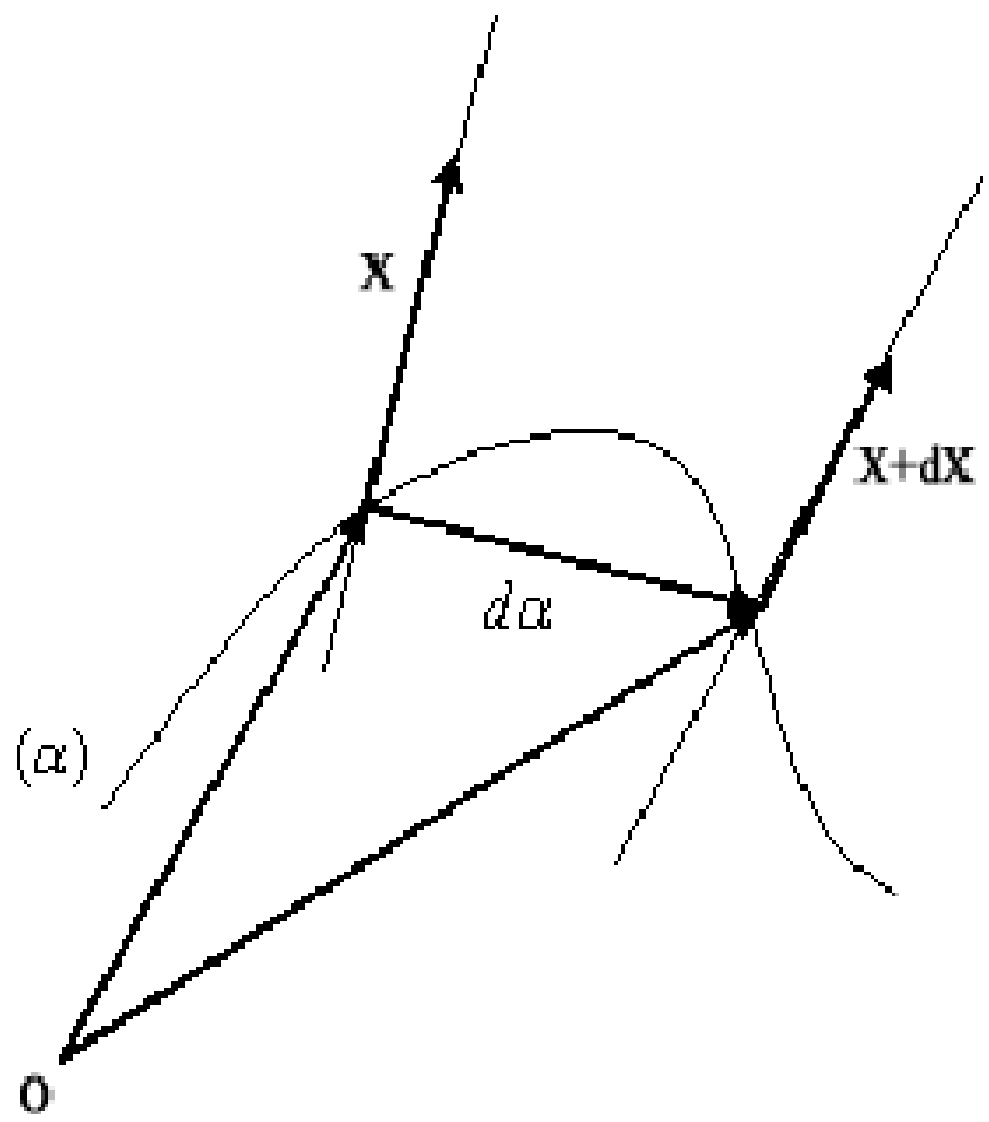

Figure 2

$$
U=\frac{X \Lambda d X}{\|X \Lambda d X\|}=\frac{X \Lambda d X}{\|d X\|} .
$$

Then, the shortest distance between the rulings $X$ and $X+d X$ is

$$
\begin{gathered}
k=\langle d \alpha, U\rangle \\
k=\frac{1}{\|d X\|}\langle d \alpha X \Lambda d X\rangle .
\end{gathered}
$$

On the other hand, the angle between the rulings $X$ and $X+d X$ is

$$
d \psi=\left\|\frac{d X}{d s}\right\| d s=\left\|D_{T} X\right\| d s .
$$

or using the equation 3.2 we get 


$$
d \psi=\sqrt{k_{g}^{2}+[I I(T, X)]^{2}} d s .
$$

By the definition 3.1 we get

$$
P_{X}=\frac{k}{d \psi}=\frac{I I(T, X)}{k_{g}^{2}+[I I(T, X)]^{2}} .
$$

Corollary 3. : Let $M$ be a ruled surface in $E^{3}$. For the distribution parameter $P_{X}$ we have

$$
P_{X}=\frac{I I(T, X)}{k_{g}^{2}+[I I(T, X)]^{2}} .
$$

Definition 2. : Let $M$ be a ruled surface in $E^{3}$. If the tangent planes of $M$ along a ruling are constants, then $M$ is called developable surface.

Theorem 4. : Let $M$ be a ruled surface in $E^{3}$. The ruled surface $M$ is developable if and only if $P_{X}=0$.

Proof. : Combining the theorem 3.1 and the definition 3.2, the proof can be easily done.

Definition 3. : The ruled surface $M$ can be given by

$$
\varphi(s, v)=\alpha(s)+v X(s), s \in I, v \in I R .
$$

If $\varphi(s+2 \pi, v)=\varphi(s, v)$ for all $s \in I$, then the ruled surface $M$ is called closed.

Definition 4. :Let $M$ be a ruled surface in $E^{3}$. A curve which meets perpendicularly each one of rulings is called an orthogonal trajectory of the ruled surface $M$.

Definition 5. : Let $M$ be a ruled surface in $E^{3}$. The foot of the common perpendicular line of the neighbouring two rulings on the main ruling is called the striction (or central) point and the locus of these points is called the striction (or central) curve of the ruled surface $M$.

Now we obtain the striction curve of the ruled surface $M$. If the distance between the striction point and the directrix of the ruled surface $M i s \bar{u}$,then the position vector can be expressed in the form

$$
\bar{\alpha}(s, \bar{u})=\alpha(s)+\bar{u} X(s) .
$$

The parameter $\bar{u}$ can be expressed in terms of the position vector of the directrix and the directed vector of the ruling. Take three neighbouring rulings of the ruled surface $M$ such that the first and second are $X$ and $X+$ 


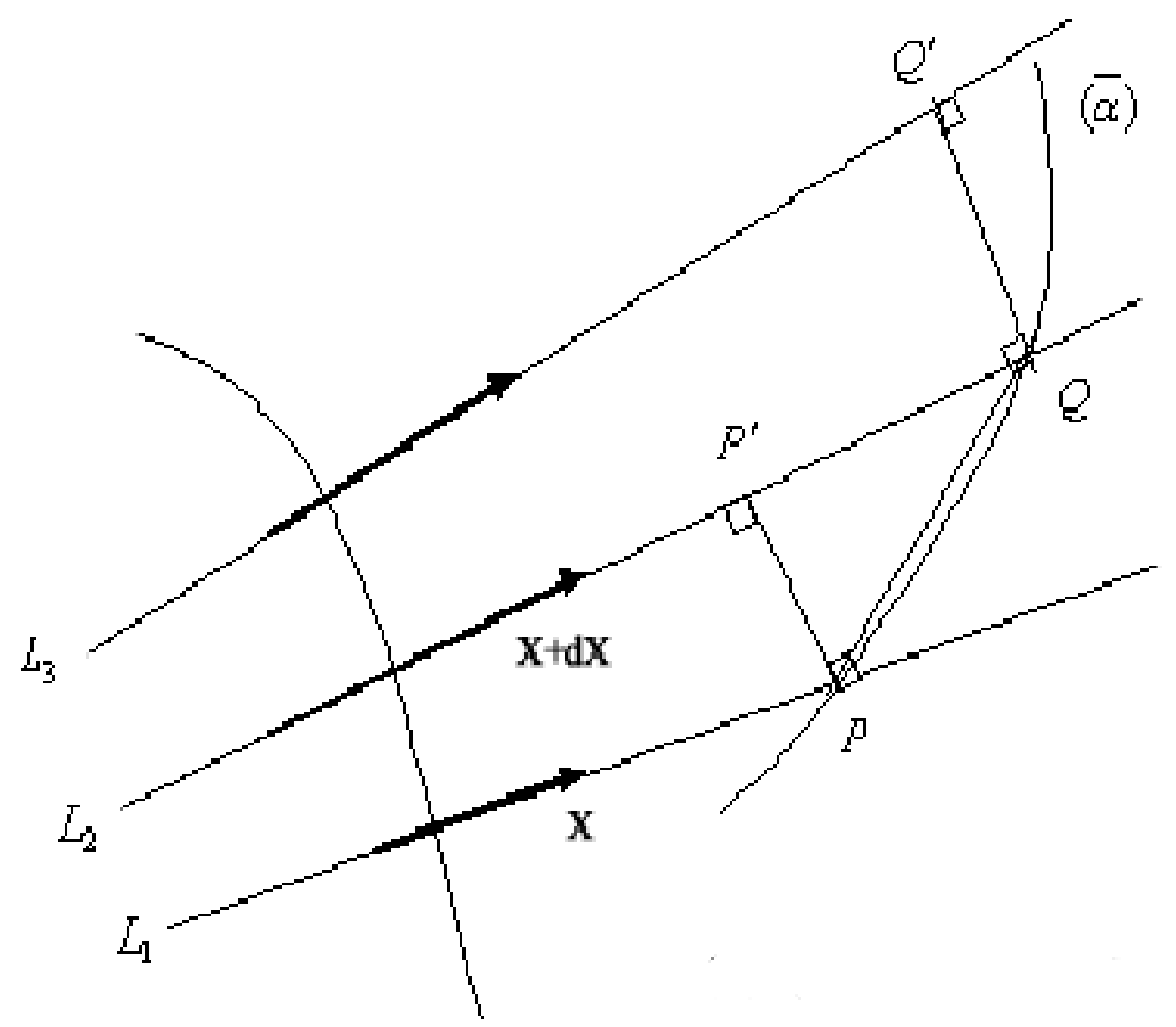

(a)

Figure 3

$d X$, respectively. Let $P, P^{\prime}$ and $Q, Q^{\prime}$ be the feet on rulings of the common perpendicular to the two rulings.

The common perpendicular vector of the rulings $L_{1}$ and $L_{2}$ is $X \Lambda d X=$ $\left(X \wedge D_{T} X\right) d s$. The vector $\overrightarrow{P Q}$ coincides with the vector $\overrightarrow{P P^{\prime}}$ in the limiting position, and $\overrightarrow{P Q}$ will be the tangent vector to the striction curve $(\bar{\alpha})$. Then we have $\langle X, \overrightarrow{P Q}\rangle=0$ and $\left\langle X+D_{T} X d s, \overrightarrow{P Q}\right\rangle=0$. From here, we get $\left\langle D_{T} X, \overrightarrow{P Q}\right\rangle=0$. Therefore we get

$$
\bar{u}=\frac{\left\langle D_{T} X, T\right\rangle}{\left\langle D_{T} X, D_{T} X\right\rangle}=\frac{k_{g}}{k_{g}^{2}+[I I(T, X)]^{2}} .
$$

Thus, the striction curve is given by 


$$
\bar{\alpha}(s)=\alpha(s)+\frac{k_{g}}{k_{g}^{2}+[I I(T, X)]^{2}} X(s) .
$$

Conversely, if $\left\langle D_{T} X, D_{T} X\right\rangle=0$, then the ruled surface $M$ is a cylinder. For the ruled surfaces, the striction curve can be taken as the directrix. Hence, we must take $\bar{u}=0$ in the eq. (3.9).

Theorem 5. : Let $M$ be a ruled surface in $E^{3}$ where $I I(T, X) \neq 0$ and given by $\varphi(s, v)=\alpha(s)+v X(s), s \in I, v \in I R$.. Then, the shortest distance between the rulings of the ruled surface $M$ along the orthogonal trajectories is the distance measured corresponding to the value $v=\frac{k_{g}}{k_{g}^{2}+[I(T, X)]^{2}}$ along the curve $\varphi_{v}: I \rightarrow M$.

Proof. :Let us consider the two rulings passing the points $\alpha\left(s_{1}\right)$ and $\alpha\left(s_{2}\right)$ where ${ }_{s} s_{1} \prec s_{2}$. The distance between the these rulings along an orthogonal trajectory is given by

$$
J(v)=\int_{s_{1}}^{s_{2}}\|A\| d s
$$

where $A=\left(1-v k_{g}\right) T+v I I(T, X) N$. From here we get

$$
J(v)=\int_{s_{1}}^{s_{2}} \sqrt{1-2 v k_{g}+k_{g}^{2} v^{2}+v^{2}[I I(T, X)]^{2}} d s
$$

To find the value of $s$ which minimizes $J(v)$, we use $J^{\prime}(v)=0$ which gives $v=\frac{k_{g}}{k_{g}^{2}+[I I(T, X)]^{2}}$. This is completed the proof.

Theorem 6. : Let $M$ be a ruled surface in $E^{3}$. Then, the point $\varphi\left(s, v_{0}\right), v_{0} \in$ $I R$, on the main ruling passing the point $\alpha(s)$ is a striction point if and only if $D_{T} X$ is the unit normal vector field of tangent plane in the point $\varphi\left(s, v_{0}\right)$ where $X$ is the tangent vector field of the directrix $\alpha$.

Proof. : Assume that the point $\varphi\left(s, v_{0}\right)$ on the main ruling passing the point $\alpha(s)$ is a striction point. We will show that $\left\langle D_{T} X, X\right\rangle=\left\langle D_{T} X, A\right\rangle=0$. If we take the derivate of the equality $\langle X, X\rangle=1$ with respect to the vector field $X$, then we obtain $\left\langle D_{T} X, X\right\rangle=0$. By direct computation the value $\left\langle D_{T} X, A\right\rangle$ we get

$$
\left\langle D_{T} X, A\right\rangle=-k_{g}+v_{0}\left(k_{g}^{2}++[I I(T, X)]^{2}\right) .
$$

Since $\varphi\left(s, v_{0}\right)$ is a striction point we can write $v_{0}=\frac{k_{g}}{k_{g}^{2}+[I I(T, X)]^{2}}$.Then, substituting the value $v_{0}=\frac{k_{g}}{k_{g}^{2}+[I I(T, X)]^{2}}$ into the above equation we obtain $\left\langle D_{T} X, A\right\rangle=0$. Thus, we can say that $D_{T} X$ normal to $X$. Because of $T_{M}\left(\varphi\left(s, v_{0}\right)\right)=\operatorname{Span}\{A, X\}$, we can write $D_{T} X \in T_{M}^{\perp}\left(\varphi\left(s, v_{0}\right)\right)$. 
Conversely, assume that $D_{T} X$ is the unit normal vector field of tangent plane in the point $\varphi\left(s, v_{0}\right)$ where $X$ is the tangent vector field of the directrix $\alpha$.

Let $A=\left(1-v_{0} k_{g}\right) T+v_{0} I I(T, X) N$ be a tangent vector field of the curve $\varphi_{v_{0}}$ : $I \times\left\{v_{0}\right\} \rightarrow M$. Since $D_{T} X$ is normal to the tangent plane $T_{M}\left(\varphi\left(s, v_{0}\right)\right)$ we get $v_{0}=\frac{k_{g}}{k_{g}^{2}+[I I(T, X)]^{2}}$.

From here we can say that the point $\varphi\left(s, v_{0}\right)$ is a striction point. This is completed the proof.

Theorem 7. : Let $M$ be a ruled surface in $E^{3}$ and $K$ be the gauss curvature function of $M$. Then, we have $K(P) \leq 0$ for all $P \in M$.

Proof. :Let $\Phi=\{X, Y\}$ be an orthonormal basis of $\chi(M)$ where $X$ is the tangent vector field of the ruling at the point $P \in M$.Thus, the matrix of the shape operator $S$ is

$$
S_{\Phi}=\left[\begin{array}{cc}
\langle S(X), X\rangle & \langle S(Y), X\rangle \\
\langle S(X), Y\rangle & \langle S(Y), Y\rangle
\end{array}\right]
$$

Since the ruling $X$ is asymptotic we get

$$
K=\operatorname{det} S_{\Phi \cdot}=-(\langle S(X), Y\rangle)^{2} \leq 0 .
$$

Theorem 8. : Let $M$ be a ruled surface in $E^{3}$. The absolute value of the Gauss curvature $K$ of the ruled surface $M$ along a ruling takes the maximum value at the striction point on this ruling.

Proof. : The set $\left\{A_{0}, X\right\}$ is an orthonormal basis of $\chi(M)$ where $A_{0}=\frac{1}{\|A\|} A$.Hence, we obtain

$$
K(s, v)==-\left(\left\langle S\left(A_{0}\right), X\right\rangle\right)^{2}=-\frac{[I I(T, X)]^{2}}{\left[1-2 v k_{g}+k_{g}^{2} v^{2}+v^{2}[I I(T, X)]^{2}\right]^{2}} .
$$

Differentiating the above equation with respect to $v$ we get

$$
\frac{\partial K}{\partial v}==4 \frac{[I I(T, X)]^{2}\left[-k_{g}+v\left(\left[k_{g}^{2}+[I I(T, X)]^{2}\right]\right)\right]}{\left[1-2 v k_{g}+k_{g}^{2} v^{2}+v^{2}[I I(T, X)]^{2}\right]^{2}} .
$$

Thus, $v=\frac{k_{g}}{k_{g}^{2}+[I I(T, X)]^{2}}$ gives us the maximum of $K(s, v)$ since

$$
\frac{\partial^{2} K}{\partial v^{2}}\left(\frac{k_{g}}{k_{g}^{2}+[I I(T, X)]^{2}}\right) \prec 0 .
$$

From here,we have 


$$
K_{\max }(v)==-\frac{k_{g}}{k_{g}^{2}+[I I(T, X)]^{2}} .
$$

On the other hand, combining the equations (3.7) and (3.10), we get

$$
K_{\max }(v)==-\left(\frac{1}{P_{X}}\right)^{2} .
$$

Then, we can say that the maximum value of the Gauss curvature $K$ of the ruled surface $M$ along ruling depends only on the distribution parameter.

\section{REFERENCES}

[1] A. HOLDITCH, Lady's and gentleman's diary for year 1858.

[2] A. B. KEMPE, A theorem in kinematics, Messenger Math. 7, 190, 1878

[3] A. B. KEMPE, Proof of the theorem in kinematics, Messenger math.8, 42,1878

[4] A. B. KEMPE, Note on Mr. Leudesdorf's theorem in kinematics, Messenger Math.7,

165-167, 1878

[5] A. B. KEMPE, Note on the theorem in kinematics, Messenger Math. 8, $130,1879$.

[6] C. LEUDESDORF, Theorem in kinematics, Messenger Math. 7, 125$127,1877$.

[7] C. LEUDESDORF, Note on the theorem in kinematics, Messenger Math. 8, 11-12, 1878.

[8] C. THAS, Properties of ruled surfaces in the Euclidean n-space, Bull. of the Instute of Math. Acad. Sinica, Vol 6Number 1, June 1978.

[9] E. B. ELLIOT, Some characteristic of kinematics on sphere, Proc. London Math. Soc. 12, 1881.

[10] H. W.GUGGENHEIMER, Differential Geometry, Mc Graw-Hill Book Company, pp. 210/338-340, (1963).

[11] H. R.MULLER, Über geschlossene bewegungsvorgange, Monatsh Math. 55, 209-214, 1951.

[12] H. R.MULLER, Erweiterung des satzes von Holditch für geschlossene Raum kurven, Abh. Braunschweig Wiss. Ges. 31, 129-135, 1980.

[13] H. R.MULLER, Verallgemeinerung einer formelvon Steiner, Abh. Braunschweig Wiss. Ges. 31, 107-113, 1978.

[14] H. POTMANN, Holditch -Sicheln, Arch, Math. 44, 373-378, 1985.

[15] H. POTMANN and J. WALLNER, Computational line geometry, Springer-Verlag, Heidelberg, 2001.

[16] H. H. HACISALİHOĞLU, On the pitch of a closed ruled surface, Mech. and Mach. Theory, Vol 7, pp.291-305, 1972. . 
[17] H. H. HACISALİHOĞLU, Diferensiyel geometri, Fen Fakültesi, Ankara, 1994

[18] H. FRANK, Ebene projektive kinematik ,Diss. Univ. Karlsruhe, 1968.

[19] J. STEINER, Ges. werke, Berlin, 1881-1882.

[20] J. HOSCHEK, Integral invarianten von regel flächen, Arch. Math. XXIV (1973),

218-224.

[21] J. TÖLKE, Eine affine verelgemeinerung eines globalen satzes von J. Steiner, Abh. Braunschweig Wiss. Ges. 30, 1-5, 1979.

[22] N. KURUOĞLU and S. KELEŞ, Properties of 2-dimensional ruled surfaces in the Euclidean space and Massey's theorem, Communications de la Faculté des Sciences de

l'Université d'Ankara, Tome 32, 1983.

[23] O. BOTTEMA, Ein problem der affinen kinematik, Konikl. Nederl. Akademie van Wetenschappen, Series A 67, 290-300, 1964 ..

[24] O. GIERING, Vorlesungen über höhere geometrie, Vieweg, BraunschweigWiesbaden , 1982.

[25] R. THÜRİNG, Studien über die Holditch'sche bewegung,Verh.Naturf. Gea Basel 67,575-594, 1956

[26] V. HLAVATY, Differentielle linien geometrie, Uitg P. Noorfhoff, Groningen,1945.

[27] W.BLASCHKE und H. R. MULLER, Ebene Kinematik, Verlag Oldenburg, München, 1956.

Received: May 31, 2006 